\title{
LARGE-SCALE INSTABILITIES IN A STOVL UPWASH FOUNTAIN
}

\author{
A. J. Saddington, P. M. Cabrita and K. Knowles \\ Aeromechanical Systems Group, Cranfield University, RMCS, \\ Shrivenham, Swindon, Wiltshire, SN6 8LA, UK
}

\begin{abstract}
The fountain flow created by two underexpanded axisymmetric, turbulent jets impinging on a ground plane was studied through the use of laser-based experimental techniques. Velocity and turbulence data were acquired in the jet and fountain flow regions using laser doppler velocimetry and particle image velocimetry. Profiles of mean and rms velocities along the jet centreline are presented for nozzle pressure ratios of two, three and four. The unsteady nature of the fountain flow was examined and the presence of large-scale coherent structures identified. A spectral analysis of the fountain flow data was performed using the Welch method. The results have relevance to ongoing studies of the fountain flow using large eddy simulation techniques.
\end{abstract}

\section{KEYWORDS}

Fountain, impinging, jet, LDV, PIV, STOVL, supersonic, turbulent, underexpanded, VSTOL

\section{NOMENCLATURE}

D nozzle exit diameter $(0.0127 \mathrm{~m})$

$h \quad$ nozzle height above ground plane

NPR nozzle pressure ratio $\left\{p_{0 c} / p_{a}\right\}$

$p_{a} \quad$ atmospheric static pressure

$p_{0 c} \quad$ settling chamber total pressure

$r \quad$ radial distance from nozzle axis

$S \quad$ nozzle spacing

$x \quad$ co-ordinate parallel to the ground plane in the plane of the jet centres (see Fig. 1)

$y \quad$ co-ordinate parallel to the ground plane in the plane of the fountain axis (see Fig. 1)

$z \quad$ co-ordinate normal to the ground plane (see Fig. 1)

$z^{\prime} \quad h-z$

$\gamma \quad$ ratio of specific heats 


\section{INTRODUCTION}

The wall jets created by the impingement on the ground of the individual jet flows from a jet-lift short take-off and vertical landing (STOVL) aircraft (with two or more nozzles) meet at a stagnation line and interact to form an upwards-flowing 'fountain' that interacts with the airframe. In some cases this can provide a beneficial lift-generating ground cushion (Anderson, 1990). The fountain flow regime may also give rise to a variety of undesirable characteristics: hot gas ingestion (HGI); ground erosion; acoustic, thermal and pressure loads on the airframe (Curtis, 2002).

Early work revealed that the fountain is sensitive to small imbalances between the jets and appears to be unstable under certain conditions (Skifstad, 1970). Further studies have reported turbulence intensities in the fountain upwash as high as $50 \%$ and a much greater rate of spreading in the fountain when compared to a free jet (Barata, 1993). Velocities normal to the axis of the fountain upwash have been found to be in the region of twenty to thirty percent of the jet exit velocity, at least for incompressible experiments (Barata, 1993; Behrouzi and McGuirk, 1993). Positioning of the fountain is largely dependent upon the momentum ratio of the wall jets with differences in their relative thicknesses causing the fountain to appear to lean (Siclari, Hill and Jenkins, 1981). Nozzle angle relative to the impingement plane and nozzle splay angle also play an important part in the fountain location and development (Siclari et al., 1981; Behrouzi and McGuirk, 1993).

Visualisation of multijet impingement has revealed the presence of large scale coherent structures, evolving from the main jets, propagating through the wall jets and dissipating in the fountain (Wohllebe and Siclari, 1978; Saripalli, 1983; Kibens, Saripalli, Wlezien and Kegelman, 1987; Cabrita, Saddington and Knowles, 2002), with possible crossover of these structures from one wall jet to the opposite side of the fountain (Childs and Nixon, 1987). This may be responsible, in part, for the large degree of spreading associated with fountain flows.

Whilst it is evident that the fountain upwash flow is unsteady, only limited data on the transient characteristics of this flow region are available. Early experiments relied on intrusive measurement techniques to provide mean pressure data (Hall and Rogers, 1969) with unsteady pressures on the ground plane being used to infer additional information (Knowles, Wilson and Bray, 1993). Techniques such as particle image image velocimetry (PIV) and laser doppler velocimetry (LDV) offer the possibility of detailed non-intrusive measurements in the fountain region. Previous investigations using these techniques have used water as the working fluid (El-Okda and Telionis, 2002) or were limited to a single nozzle pressure ratio (Elavarasan, Venkatakrishnan, Krothapalli and Lourenço, 2000).

This paper reports on PIV and LDV measurements of the three-dimensional fountain flow-field generated by the impingement of two axisymmetric, compressible, under-expanded, turbulent jets on a ground plane.

\section{FACILITIES AND INSTRUMENTATION}

\section{Impinging jet facility}

The experiments were conducted in a dedicated impinging jet facility at Shrivenham. A schematic of the twin impinging jet flow field is shown in Figure 1. The test rig consisted of a small cylindrical settling chamber with an internal diameter of $230 \mathrm{~mm}$ and a height of $210 \mathrm{~mm}$. It has two internal screens and removable nozzle mounting plates that enable the configuration of various nozzle spacing and splay angles. Air is supplied to the settling chamber by two Howden screw-type compressors capable of a maximum flow rate of $0.9 \mathrm{kgs}^{-1}$ at pressures of up to 7 bar gauge. The pressure in the settling chamber was adjusted using a pneumatic CompAir A119 computer-controlled valve that was capable of maintaining the nozzle pressure ratio (NPR) to within $\pm 0.2 \%$ of the desired value. The settling chamber 
was instrumented with a thermocouple and pressure transducer that provide information on the stagnation conditions.

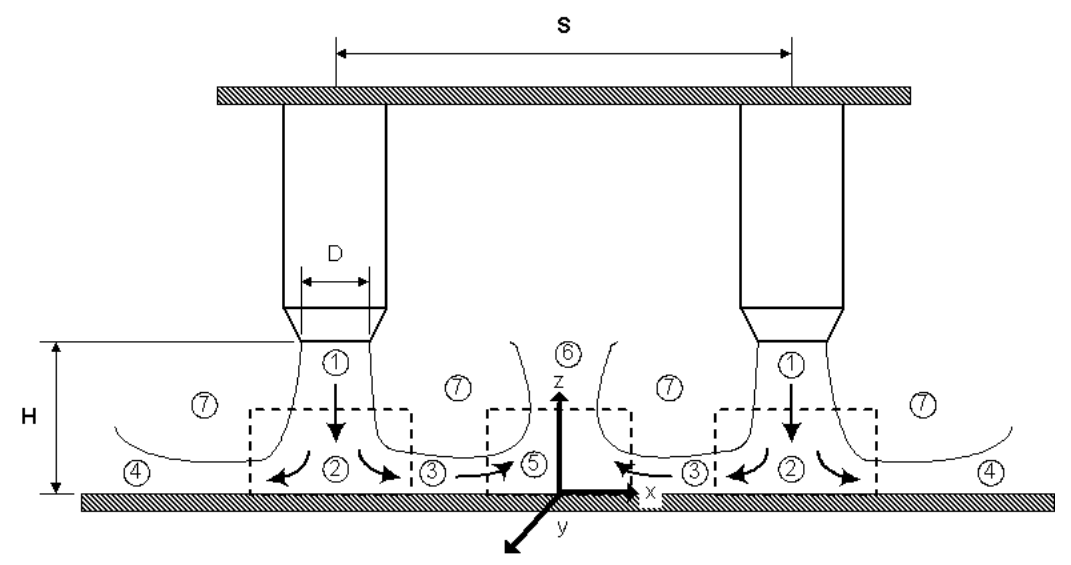

1. Lift jet flow

2. Jet impingement region

3. Inner wall jet flow

4. Outer wall jet flow

5. Fountain formation region

6. Fountain upwash flow

7. Entrainment

Figure 1: Schematic of the experimental set-up illustrating the main flow characteristics.

\section{PIV}

The PIV equipment consisted of a New Wave Gemini Nd:YAG double-pulsed laser which, through the use of a combination of spherical and cylindrical lenses, created a light sheet approximately one millimetre thick, positioned perpendicular to the impingement plane and passing through the plane defined by the nozzle axes. The PIV double-pulsed image pairs were acquired using a Kodak Megaplus ES1.0 digital camera with a maximum resolution of $1008 \times 1016$ pixels at a rate of $15 \mathrm{~Hz}$. The camera was fitted with a $60 \mathrm{~mm}$ (f2.8) Nikon lens and placed normal to the light sheet. This allowed for a maximum field of view of $60 \mathrm{~mm} \times 30 \mathrm{~mm}$, giving a resolution of $81 \mu \mathrm{m}$ per pixel. The time separation between the two laser pulses was varied between $2.6 \mu \mathrm{s}$ and $6.6 \mu \mathrm{s}$ according to the calculated isentropic jet exit velocity for the particular NPR. The jets were seeded using JEM Hydrosonic long-lasting fluid droplets of approximately $1 \mu \mathrm{m}$ diameter generated by a TSI 9306 Six-jet atomizer connected to a Clarke compressor. The ambient air was seeded with smoke particles produced by a Le Maitre Turbo Mist fog generator.

\section{$L D V$}

LDV measurements were made using a Dantec system consiting of a Lexel Model 95 water-cooled Argonion laser, a Dantec $60 \times 41$ transmitter with $60 \times 24$ fibre optic manipulators, $57 \mathrm{~N} 20$ burst spectrum analyser, a 2D FiberFlow probe and a $1 \mathrm{~m}$ focal length lens with a $2 \times$ beam expander. The lens and beam expander combination created a measurement volume of $0.15 \mathrm{~mm} \times 4.2 \mathrm{~mm} \times 0.15 \mathrm{~mm}$. Alignment was completed using a $35 \mu \mathrm{m}$ pinhole with a photovoltaic cell. Time-averaged LDV data was derived from a sample size of 10000. Dantec Burstware data processing software was used to export the time series data, that was further processed using MATLAB.

\section{METHODOLOGY}

The configuration used for the present study comprised two identical $63.5 \mathrm{~mm}$ long axisymmetric convergent nozzles with an exit diameter, $D$ of $12.7 \mathrm{~mm}$. The distance between the nozzle centres, $S$ was $88.9 \mathrm{~mm}(7 \mathrm{D})$. The ground plane consisted of a one metre square, $10 \mathrm{~mm}$ thick, aluminium plate. The distance between the nozzle exits and the impingement plane was varied between $2.4 D$ and 8.4D. Data were recorded for NPRs between 1.05 and 4 . 
Approximately 500 PIV image pairs were acquired to determine the mean flow, and first order flow statistics. The commercial software, Insight v3.3, developed by TSI was used to analyse the images. A cross-correlation algorithm was used to process the images and extract the instantaneous vector field. Interrogation windows of $32 \times 32$ pixels were employed in the processing. The size of the interrogation window was chosen to allow for a minimum of 10 seeding particles per interrogation area and to allow for the maximum in-plane particle displacement to be less than one quarter of the size of the interrogation window (Keane and Adrian, 1990). Inherent to PIV processing are the spurious vectors, which on average, accounted for less than $3 \%$ of the total. They were removed using a pass-band filter followed by a local median filter. The resulting empty spaces were filled with interpolated values from the surrounding area.

LDV data was acquired in two regions: in the jet along a line described by the nozzle axis and in the fountain along a line joining the nozzle centres at heights of $z / D=0.5, z / D=1$ and $z / D=2$.

\section{RESULTS AND DISCUSSION}

Results are presented for the twin impinging jet experiment described above. Data will be presented for NPR $=3$ at a range of nozzle height to diameter ratios. The discussion is divided into two parts: the jet flow and the fountain flow.

\section{Jet flow}

The underexpanded impinging jet flow field can be divided into three main regions (Donaldson and Snedeker, 1971): the free jet region, where the flow is primarily inviscid and contains the series of expansion and compression waves; the impingement region, which is characterised by strong gradients that alter significantly the local flow properties; the wall jet, which consists of a radial redirection of the jet flow after impingement.

Figure 2 shows the centreline mean and rms axial velocity as a function of distance from the nozzle exit plane, $z^{\prime}$ ( $z^{\prime}=h-z$, where $z$ is the ground-normal coordinate) for NPRs of two, three and four. The plots are superimposed onto time-averaged schlieren images of the experiment to the same scale. Spatial correlation of the LDV data with the schlieren images is very good. At an NPR of two, there is some acceleration of the flow as it leaves the nozzle exit and some evidence of a shock structure, although it is very weak. The rms velocity initially falls but then rises again as the potential core starts to decay. At the higher NPRs the expansion and recompression through the shock structure is clearly evident. The mean velocity data agree well with previous free jet experiments (Saddington, Lawson and Knowles, 2004) up to $z^{\prime} / D \approx 5-6$ after which point the shock cells appear to shorten as impingement approaches. In general, the rms velocity component increases with distance from the nozzle exit plane, varying through the shock structure approximately in phase with the mean velocity fluctuations. Similar fluctuations in rms velocity were observed when the jets were operated without a ground plane.

Figure 3(a) shows PIV-derived velocity magnitude contours with superimposed streamlines at $h / D=2.4$. The effect of the presence of the fountain is clearly visible in the entrainment process occurring on both sides of the jet. The left hand side (outer side) streamlines display a continuous entrainment of ambient air along the jet shear layer and wall jet. The right hand side (inner side) displays a different pattern. This is due to the presence of the fountain where the pressure is sub-atmospheric (Abbott and White, 1989), inducing a higher velocity in the fountain-facing wall jet (inner side) and a thickening of the inner shear layer of the jet.

It is difficult to determine with absolute certainty the accuracy of the PIV measurements because detailed velocity field data are not available in the literature for this flow field. A comparison of PIV-derived Mach number data with a simple shadowgraph (Figure 3(b)) indicates, however, that the PIV has been able to capture the location of the important features of the underexpanded impinging jet. 


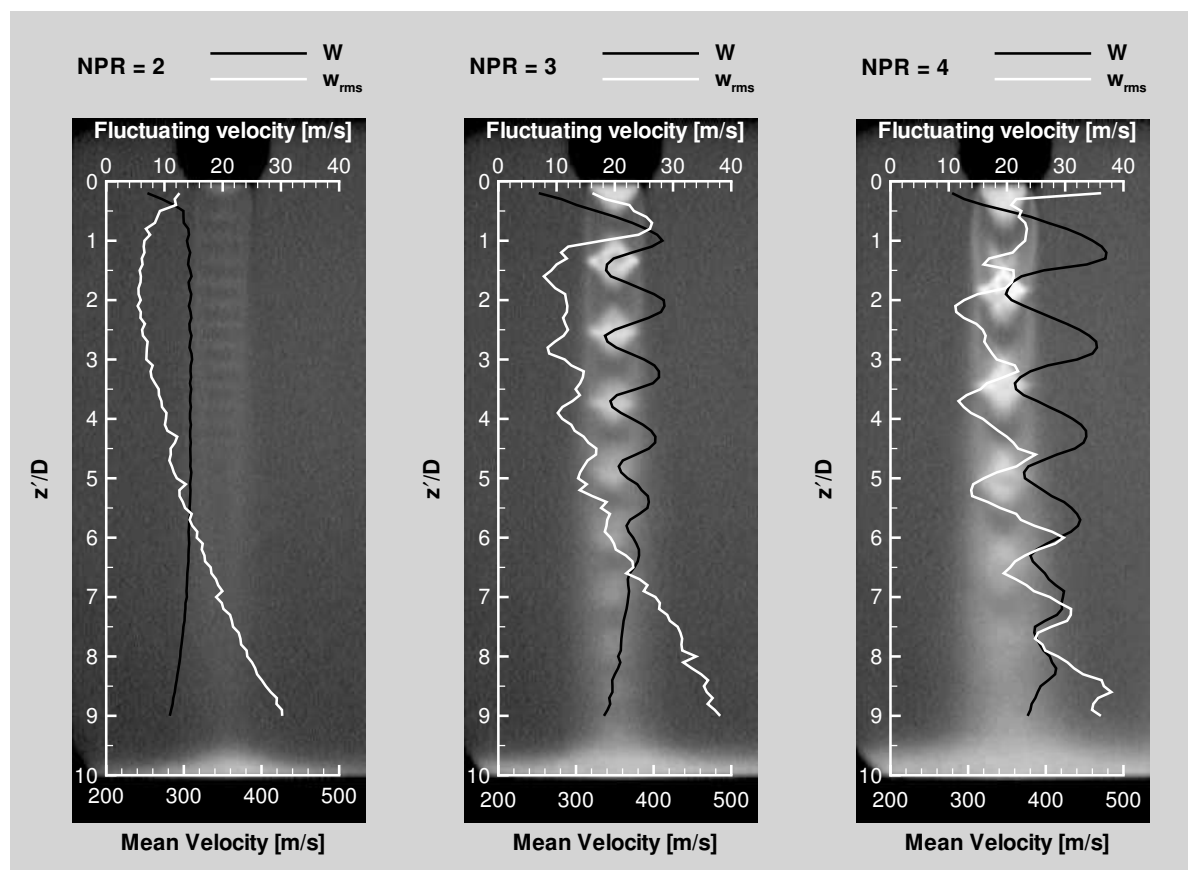

Figure 2: Centreline mean and rms axial velocity for an impinging jet $(h / D=10, \mathrm{NPR}=3)$ superimposed on time-averaged schlieren images to the same scale.

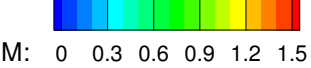

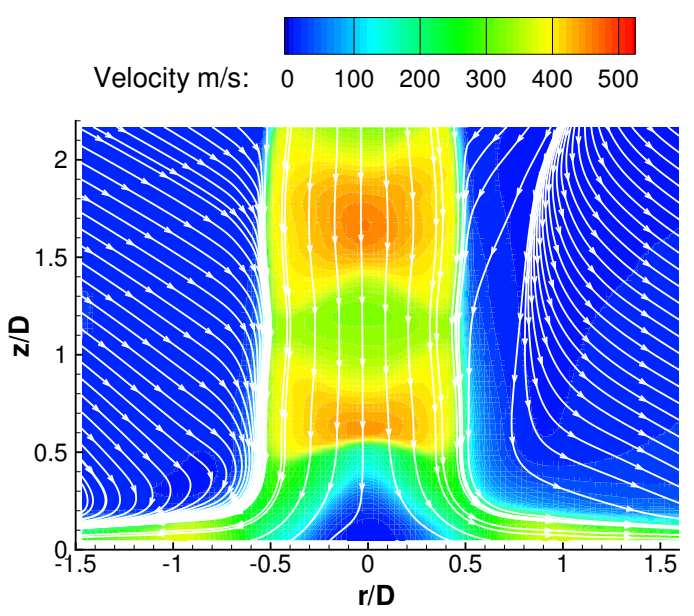

(a) Contours of velocity magnitude with superimposed streamlines.

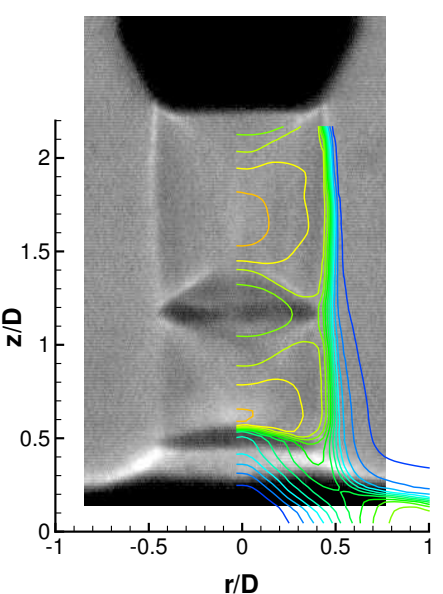

(b) Contours of Mach number superimposed onto a shadowgraph image.

Figure 3: PIV-derived images of the left-hand-side jet $(h / D=2.4, \mathrm{NPR}=3)$. 


\section{Fountain flow}

Figure 4 shows two sequential instantaneous velocity magnitude contours at a non-dimensional nozzle height of $h / D=4.4$. The images are separated temporally by $67 \mathrm{~ms}$. The behaviour of the fountain flow is quite different from the jet flow in that the instantaneous velocity fields do not correlate well with the time-averaged one (Figure 5) - indicating that it is a highly unsteady flow. This unsteadiness results from the collision of two wall jets that contain vortical structures and are themselves highly turbulent. The instantaneous velocity fields show a high degree of asymmetry, the presence of large-scale vortical structures and a stagnation region whose location has been observed to vary randomly. Although the instantaneous fountain flow is somewhat incoherent, it is clear from the images (Figure 4) that it is inclined relative to the vertical. Through the observation of a sequence of these instantaneous velocity fields it appears that the fountain inclination is related to the strength and location of the dominant vortical structures. Unfortunately, the frame rate at which these data were acquired does not allow for the temporal resolution of these structures.

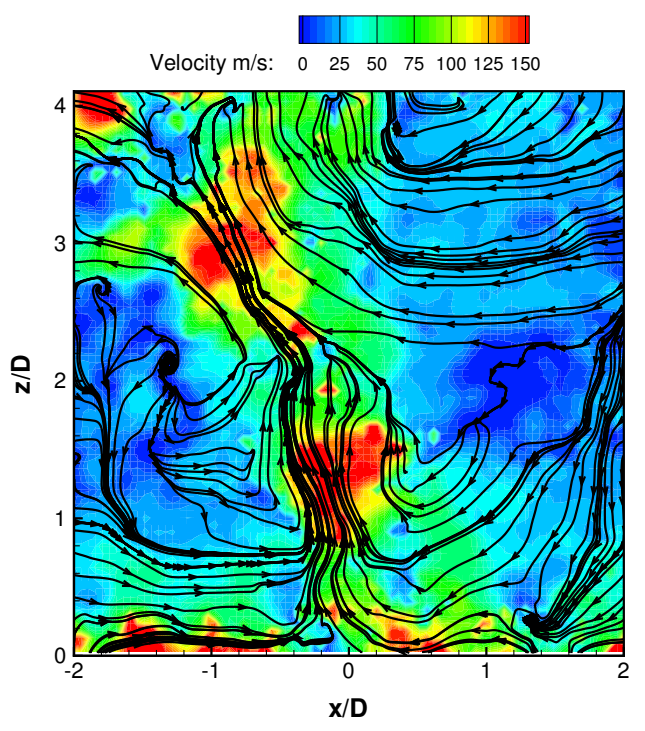

(a) time $=t$.

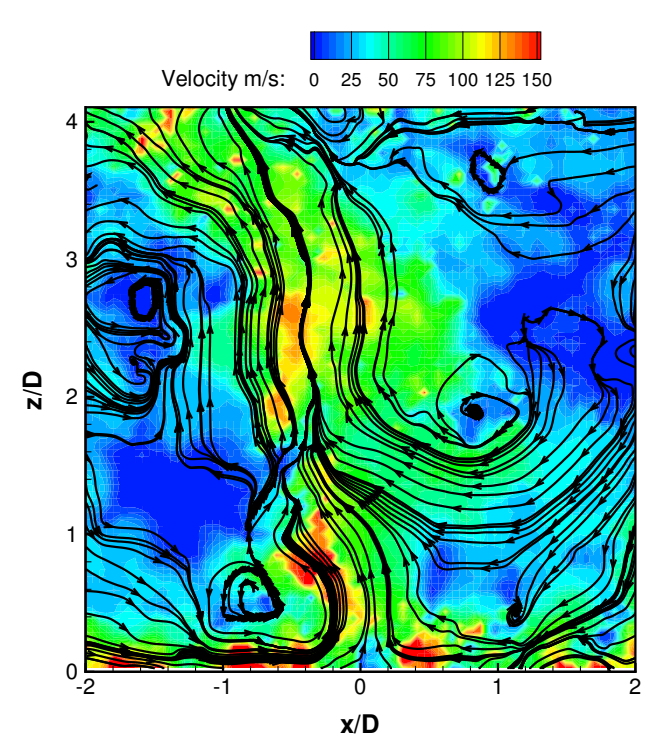

(b) time $=t+67 \mathrm{~ms}$.

Figure 4: Instantaneous velocity magnitude contours of the fountain flow $(h / D=4.4, \mathrm{NPR}=3)$.

Figure 5 shows the mean velocity magnitude contours for two nozzle heights, $h / D=4.4$ and $h / D=6.4$ at an NPR of 3 and reveals two well-defined recirculation regions formed between the fountain and impinging jet flows. At the lower height (Figure 5(a)) there is a recirculation zone to the left of the fountain, which is inclined to that side. A second recirculation zone is partially visible in the top right hand corner of this figure, however, it is the left-hand vortex which appears to dominate the flow. At a slightly higher height (Figure 5(b)) the flow is more symmetrical. Two recirculation zones are clearly visible centred laterally almost exactly midway between the fountain axis and the jet axis. The recirculation zone on the right-hand side is positioned lower than the one on the left and as a consequence, the fountain is inclined to the right. The fountain appears to be a bi-stable flow which, with symmetrical geometry and jet conditions, in the mean, would produce a fountain with no inclination. The time-averaged velocity contours were, therefore, quite surprising. The sample size of 500 is not large but should be sufficient to give a good representation of the mean. Further investigation showed the fountain inclination to be most likely dependent upon asymmetries in the geometry of the experimental rig. This was confirmed by swapping the nozzles over which, despite there being no measureable difference in geometry, changed the fountain inclination direction. These observations confirm the sensitivity of the fountain to small 
imbalances in system (Skifstad, 1970), which has also been reported in other recent experiments on twin-jet fountain flows (Elavarasan et al., 2000; El-Okda and Telionis, 2002).

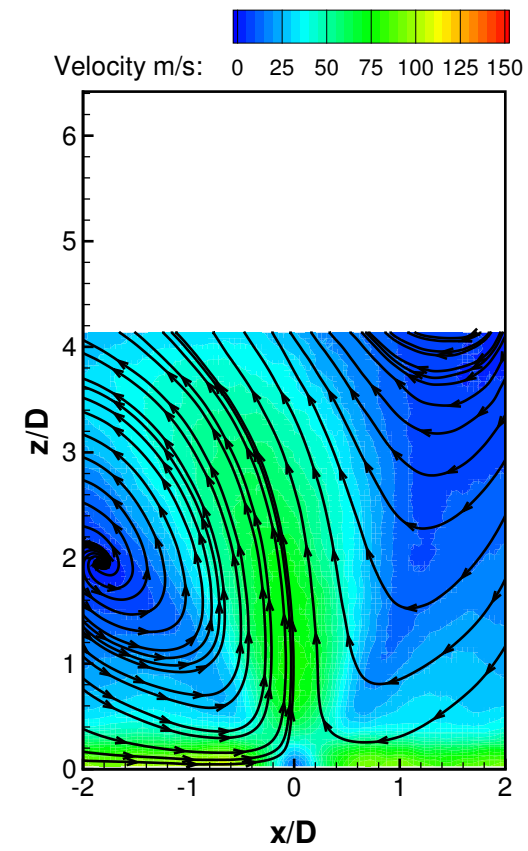

(a) $h / D=4.4$.

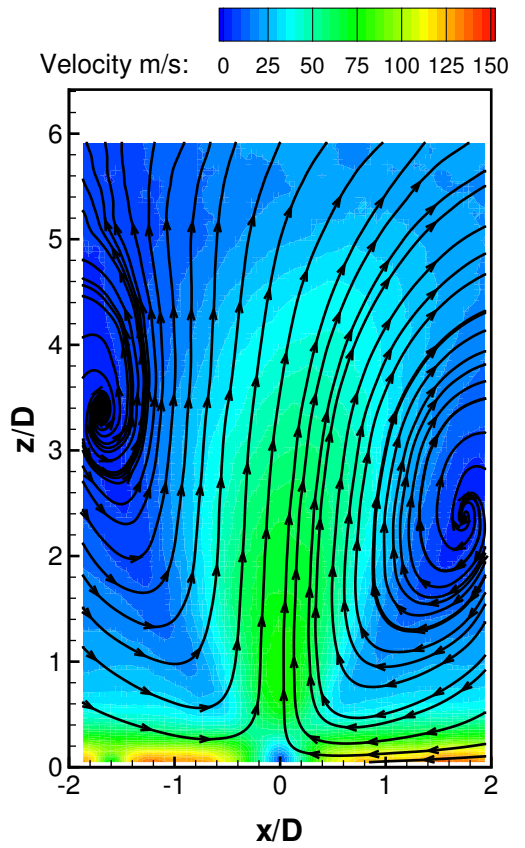

(b) $h / D=6.4$.

Figure 5: Time-averaged velocity magnitude contours of the fountain flow (NPR $=3$ ).

Figure 6(a) shows the instantaneous vertical, $w$ velocity profiles along the geometric fountain axis (i.e. $x / D=0$ ) for two sequential instants in time. Also shown in Figure 6(a) is the mean profile. The instantaneous profiles clearly show the unsteady nature of the fountain. The upwash velocity shows instantaneous values of up to $160 \mathrm{~ms}^{-1}$, approximately double the peak mean value, which is attained at $z / D \approx 0.7$. The point at which the vertical velocity is zero at $z / D=0$ indicates the location of the fountain stagnation line. Only one of the instantaneous profiles shows a vertical velocity close to zero at $z / D=0$, which supports the previous observation that the fountain stagnation line moves from one time instant to another. The mean profile shows a rapid increase from approximately zero velocity to its peak at $z / D \approx 0.7$. The velocity then decreases asymptotically towards zero as vertical velocity decays. It is interesting to note that for the nozzle heights tested (Figure 6(b)) the mean vertical velocity profile is independent of nozzle height. The slight difference for the $h / D=4.4$ case is a consequence of the large fountain inclination (see Figure 5(a)) producing a vertical velocity lower than expected.

The irregular time-spaced LDV data was resampled at two times the mean data rate using a nearest neighbour resampling technique (Broersen, 1999). Figure 7 shows LDV histograms of the $x$-component of velocity at NPR $=3, h / D=4.4$ and $z / D=1$. Either side of the fountain centre (figures 7 (a) and 7(c)) the histograms present a skewed distribution typical of flows with strong gradients of turbulence intensity. At the centre of the fountain (Figure 7(b)), the histogram displays two peaks, clearly indicating an oscillation.

For the spectral analysis around 200000 samples were used. These were divided into segments of $2^{11}$ samples and processed using the Welch method and a Hanning window in order to reduce the spectral leakage. The Welch method splits a set of data into smaller sets and calculates the periodogram of each set. The frequency domain coefficients arising from calculating the periodograms are averaged over the frequency components of each data set. This results in a power spectrum that is a smoothed version of 


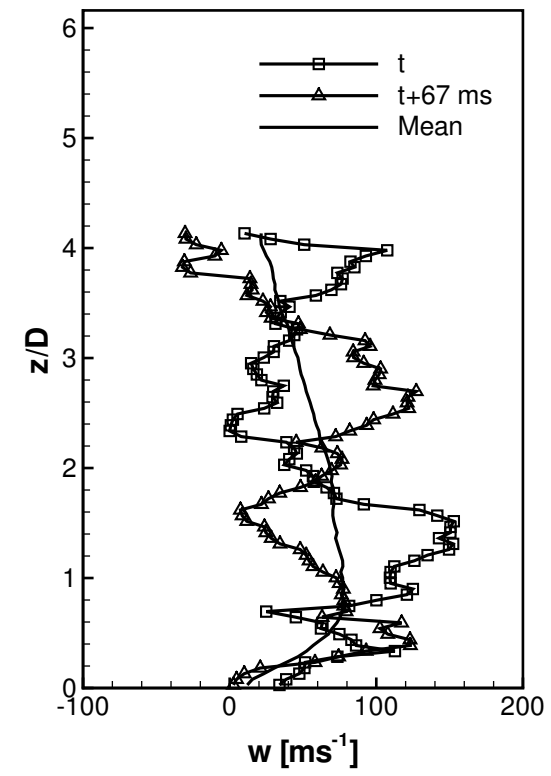

(a) $h / D=4.4$.

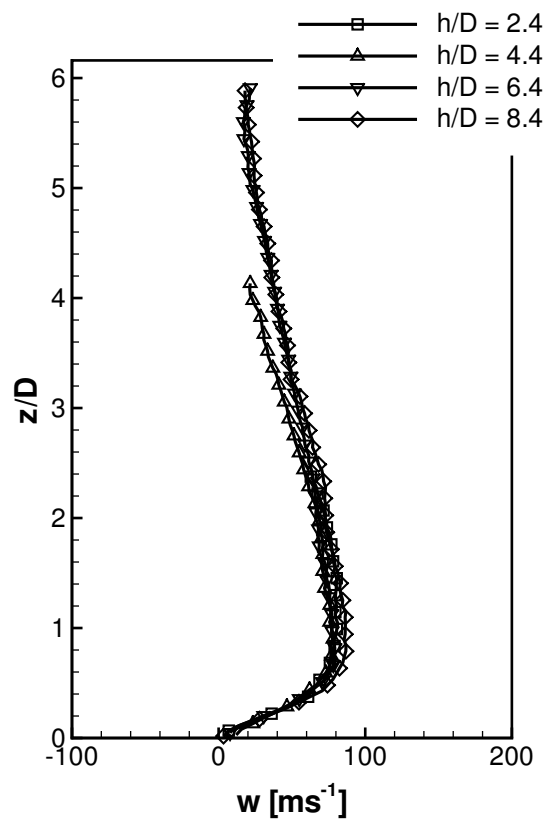

(b) Mean profiles for all heights.

Figure 6: Vertical velocity profiles along the geometric fountain axis $(\mathrm{NPR}=3)$.

the original, with less noise. In this way, the Welch method enables low pass filtering of the data.

The normalised power spectral density is shown as a function of frequency in Figure 8 for three nozzle heights and two measurement heights at an NPR of 3. In general, the frequency spectra is somewhat unremarkable with the exception of the data for $h / D=4.4, z / D=1$ where a clear peak is seen in the normalised power spectral density at approximately $240 \mathrm{~Hz}$ indicating that under these conditions the fountain does appear to exhibit a characteristic frequency. Fountain flow instabilities have previously been observed to occur at similar non-dimensional nozzle heights (Elavarasan et al., 2000) although no attempt was made to quantify the frequency.

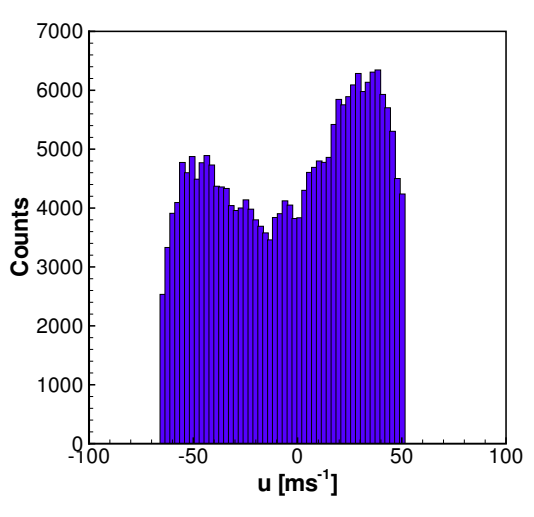

(a) $0.1 D$ left of fountain.

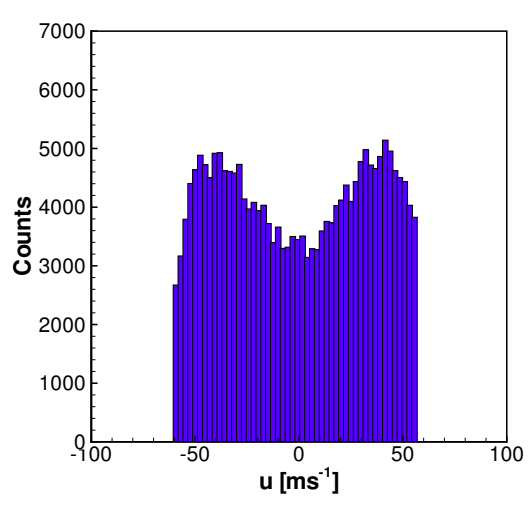

(b) Centre of fountain.

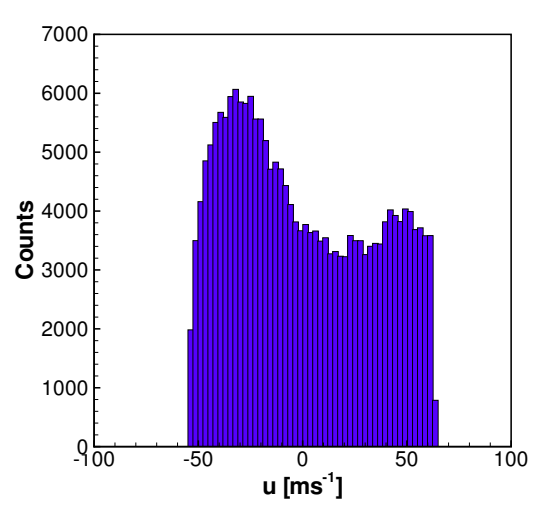

(c) $0.1 \mathrm{D}$ right of fountain.

Figure 7: Distribution of sampled velocity in the fountain, $(\mathrm{NPR}=3, h / D=4.4, z / D=1)$. 


\section{CONCLUSIONS}

An experimental study was carried out into the fountain flow created by two underexpanded axisymmetric, turbulent jets impinging on a ground plane. The entrainment characteristics of the jets were found to be altered by the presence of the fountain, which is shown to be a highly unsteady flow. Instantaneous velocity fields of the fountain show a high degree of asymmetry, the presence of large-scale vortical structures and a stagnation region whose location was observed to vary randomly. The mean vertical velocity profile through the fountain is shown to be independent of nozzle height. Spectral analysis of the laser doppler velocimetry data identified a characteristic frequency in the fountain of approximately $240 \mathrm{~Hz}$ at a nozzle height of 4.4 diameters and a measurement height of 1 diameter.

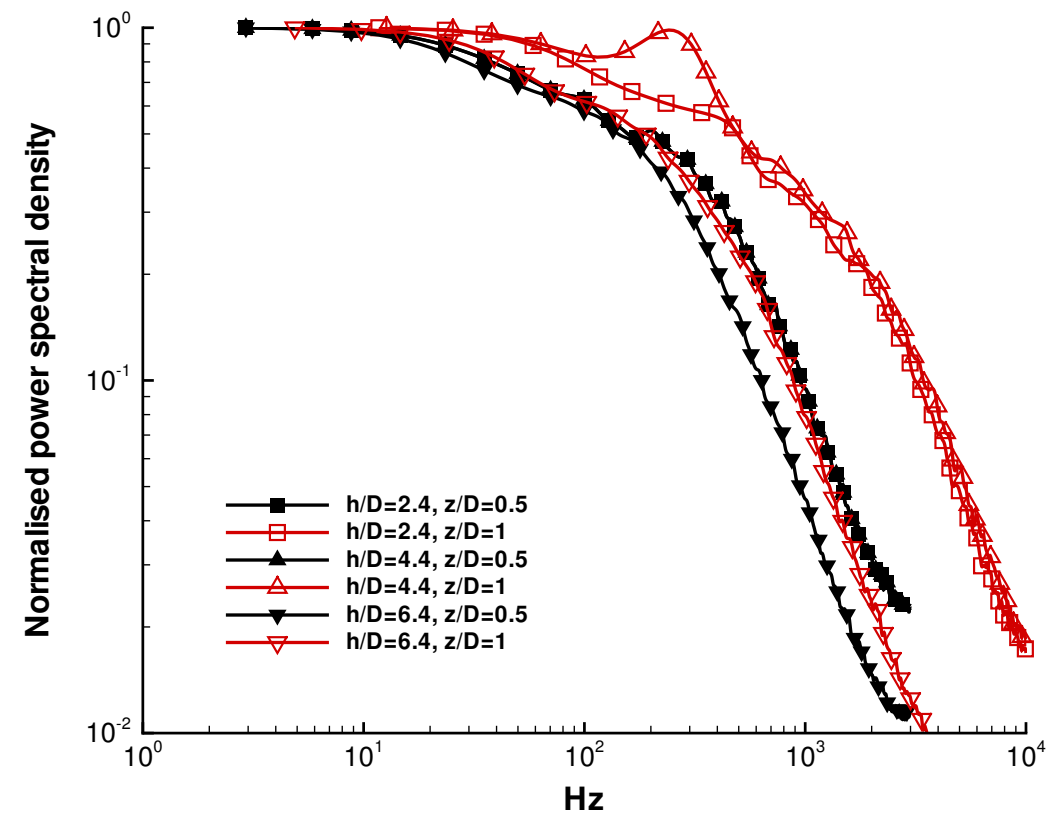

Figure 8: Frequency spectra in the fountain, $(\mathrm{NPR}=3)$.

\section{ACKNOWLEDGEMENTS}

This work was partially funded by the Engineering and Physical Sciences Research Council under grant GR/R42894/01 and their support is gratefully acknowledged.

\section{REFERENCES}

Abbott, W. A. and White, D. R. (1989), The effect of nozzle pressure ratio on the fountain formed between two impinging jets, Technical Memorandum P1166, RAE.

Anderson, S. B. (1990), Jet-powered v/stol aircraft - lessons learned, in 'International Powered Lift Conference and Exhibit', London, UK, pp. II.1.1-II.1.14.

Barata, J. M. M. (1993), 'Fountain flows produced by multi-jet impingement on a ground plane', AIAA Journal of Aircraft 30(1), 50-56. 
Behrouzi, P. and McGuirk, J. J. (1993), Experimental data for cfd validation of impinging jets in crossflow with application to astovl flow problems, in 'AGARD Conference Proceedings CP-534, Fluid Dynamics Panel Symposium', Winchester, UK.

Broersen, P. M. T. (1999), The performance of spectral quality measures, in '16th IEEE Instrumentation and Measurement Technology Conference’, Venice, Italy, pp. 751-756.

Cabrita, P. M., Saddington, A. J. and Knowles, K. (2002), Unsteady features of twin-jet stovl ground effects, in 'International Powered Lift Conference and Exhibit', Williamsburg, VA, USA. Paper no. 2002-6014.

Childs, R. E. and Nixon, D. (1987), Turbulence and fluid/acoustic interaction in impinging jets, in 'International Powered Lift Conference and Exhibit', Santa Clara, CA, USA, pp. 447-458. SAE paper 872345 .

Curtis, P. (2002), A review of the status of ground effect/environment technologies, in 'International Powered Lift Conference and Exhibit', Williamsburg, VA, USA. Paper no. 2002-5985.

Donaldson, C. D. and Snedeker, R. S. (1971), 'A study of free jet impingement. part 1. mean properties of free and impinging jets', Journal of Fluid Mechanics 45(2), 281-319.

El-Okda, Y. and Telionis, D. P. (2002), Experimental investigation of twin jet impinging on the ground with and without a free stream, in 'International Powered Lift Conference and Exhibit', Williamsburg, VA, USA. Paper no. 2002-5976.

Elavarasan, R., Venkatakrishnan, L., Krothapalli, A. and Lourenço, L. (2000), Supersonic twin impinging jets, in '38th Aerospace Sciences Meeting and Exhibit', Reno, NV, USA. Paper no. 2000-0812.

Hall, G. R. and Rogers, K. H. (1969), Recirculation effects produced by a pair of heated jets impinging on a ground plane, Contractor Report CR-1307, NASA.

Keane, R. D. and Adrian, R. J. (1990), 'Optimization of particle image velocimeters', Measurement Science and Technology 1(11), 1202-1215.

Kibens, V., Saripalli, K. R., Wlezien, R. W. and Kegelman, J. T. (1987), Unsteady features of jets in lift and cruise modes for vtol aircraft, in 'International Powered Lift Conference', Santa Clara, CA, USA, pp. 543-552. SAE paper 872359.

Knowles, K., Wilson, M. J. and Bray, D. (1993), 'Unsteady pressures under impinging jets in cross-flows', AIAA Journal 31(12), 2374-2375.

Saddington, A. J., Lawson, N. J. and Knowles, K. (2004), 'An experimental and numerical investigation of under-expanded turbulent jets', The Aeronautical Journal 108(1081), 145-152.

Saripalli, K. R. (1983), 'Visualization of multijet impingement flow', AIAA Journal 21(4), 483-484.

Siclari, M. J., Hill, W. G. and Jenkins, R. C. (1981), 'Stagnation line and upwash formation of two impinging jets', AIAA Journal 19(10), 1286-1293.

Skifstad, J. G. (1970), 'Aerodynamics of jets pertinent to vtol aircraft', Journal of Aircraft 7(3), 193-204.

Wohllebe, F. A. and Siclari, M. J. (1978), 'Fountain and upwash flowfields of multijet arrangements', Journal of Aircraft 15(8), 468-473. 